\title{
MicroRNA-16 Promotes Ovarian Granulosa Cell Proliferation and Suppresses Apoptosis Through Targeting PDCD4 in Polycystic Ovarian Syndrome
}

\author{
Xiafei Fu Yuanli He $^{\mathrm{a}}$ Xuefeng Wang ${ }^{\mathrm{a}}$ Dongxian Peng ${ }^{\mathrm{a}} \quad$ Xiaoying Chen $^{\mathrm{a}}$ \\ Xinran Lia Qing Wan ${ }^{a}$ \\ aDepartment of Obstetrics and Gynecology, Zhujiang Hospital of Southern Medical University, \\ Guangzhou, China
}

\section{Key Words}

Mir-16 • Granulosa cells • PDCD4 • Testosterone • Polycystic ovary syndrome

\begin{abstract}
Background/Aims: Several miRNAs have been reported to be involved in the pathogenesis of polycystic ovarian syndrome (PCOS). However, the biological roles of miR-16 and its molecular mechanisms in PCOS development remain to be elucidated. Methods: qRT-PCR was performed to detect the expression levels of miR-16 and programmed cell death protein 4 (PDCD4). GCs proliferation, cell cycle distribution and apoptosis were examined by MTT assay and flow cytometry analysis. Luciferase reporter assay and RIP assay were applied to confirm the regulatory relationship between miR-16 and PDCD4. Western blot was applied to measure the protein levels of PDCD4, PCNA and caspase-3. ELISA kits were used to determine the serum levels of steroids. Results: miR-16 expression was down-regulated in ovarian cortex tissues and serums of PCOS patients. PDCD4 expression was up-regulated in ovarian cortex tissues of PCOS patients. miR-16 overexpression facilitated cell proliferation, induced cell cycle progression, and inhibited apoptosis in GCs. Moreover, PDCD4 was a direct target of miR-16. Also, enforced expression of PDCD4 abated the effects of miR-16 on GCs growth and apoptosis. Additionally, testosterone resulted in a decrease of miR-16 expression and an increase of PDCD4 expression, thus blocking cell growth and enhanced apoptosis in GCs. Furthermore, miR-16 overexpression alleviated PCOS in vivo by regulating PDCD4. Conclusions: miR-16 promoted ovarian GCs proliferation and inhibited apoptosis through directly targeting PDCD4 in PCOS, contributing to a better understanding of the molecular mechanism of GCs dysregulation and providing a promising target in PCOS.

(C) 2018 The Author(s)

Published by S. Karger AG, Basel
\end{abstract}

\section{Introduction}

Polycystic ovary syndrome (PCOS) is a common heterogeneous endocrine disorder and metabolic disturbance among female individuals, with an estimated incidence of 7\%-9\% or 
more in women of reproductive age worldwide [1]. The manifestations of PCOS generally include oligomenorrhea or amenorrhea, abnormal folliculogenesis, chronic anovulation, hyperandrogenemia, and even infertility [2]. PCOS is also associated with insulin resistance, obesity, dyslipidemia, hypertension, pancreatic $\beta$-cell dysfunction, increasing the risk of type 2 diabetes mellitus (T2DM) and cardiovascular diseases [3, 4]. Genetic basis, environmental factor, chronic inflammation, oxidative stress, and lifestyle have been reported to be involved in the occurance and development of PCOS $[5,6]$. At the early phases of follicle development, atresia is triggered by oocyte apoptosis followed by death of the surrounding granulosa cells (GCs), which provide the oocytes with nutrients and growth regulators [7]. Several studies have demonstrated that dysregulation of GCs may be responsible for the abnormal folliculogenesis and excess production of intraovarian androgens in women with PCOS [8]. Therefore, it is clinically crucial to explore the pathogenetic mechanisms underlying GCs dysfunction in PCOS patients for identifying novel diagnostic and therapeutic biomarkers.

microRNAs (miRNAs), a class of small non-coding RNA molecules with 21-25 nucleotides in length, have emerged as important regulators of gene expression at the posttranscriptional level by binding to the 3' untranslated regions (3'UTR) of target mRNAs, thus causing mRNA degradation or translational repression. Previous studies elucidate that miRNAs play critical regulatory roles in a variety of developmental and physiological processes, such as cell proliferation, development, survival, apoptosis, and tumorigenesis [9]. Aberrant expression of miRNAs is reported to be associated with the progression of various diseases, including PCOS [10-12]. miR-16, located in 13q14 chromosome, was initially found to be related to chronic lymphocytic leukemia [13]. Subsequent studies reveal that miR-16 is frequently down-regulated and plays a tumor suppressive role by affecting cell proliferation, cell cycle, invasion, and apoptosis in different types of malignancies, such as breast cancer, lung cancer, and prostate cancer [14-16]. Of particular interest, a previous report discovers that serum expression of miR-16 is decreased in PCOS patients [17]. However, the biological roles of miR-16 and its possible molecular mechanisms in PCOS progression are far from being elucidated.

In the present study, we demonstrated that miR-16 overexpression promoted cell proliferation, induced cell cycle progression and diminished apoptosis of GCs in PCOS by down-regulating programmed cell death protein 4 (PDCD4). We also found that testosterone down-regulated miR-16 and up-regulated PDCD4, thereby inhibiting GCs growth and promoted apoptosis. Our findings provide new evidence for the regulatory roles of miRNAs in PCOS and suggest that miR-16 may be a promising molecular target for GCs dysfunction in PCOS therapy.

\section{Materials and Methods}

\section{Clinical samples}

The lesion ovarian cortex tissue samples were collected from 19 women patients diagnosed with PCOS and hospitalized from December 2013 to November 2014. The diagnosis of PCOS was confirmed according to Rotterdam 2003 criteria [2]. Normal ovarian cortex tissue samples were collected from 19 control healthy women without hirsutism, androgen excess, ovulatory dysfunction, with a history of predictable regular menstrual cycles. All patients had not taken hormonal drugs, such as contraceptives, antiandrogenic agents and insulin sensitizers, to interfere with sex hormone secretion and metabolism, for 6 months prior to enrolling in the study, and none were pregnant. All protocols used in this study were approved by the Institutional Ethics Committee of Zhujiang Hospital of Southern Medical University. The signed informed consents were obtained from all participants.

Isolation, culture and treatment of human GCs

Isolation of human GCs was performed as previously described [18]. Briefly, the ovaries were harvested from PCOS patients and healthy controls that undergo their first in vitro fertilization embryo transfer, and then GCs were obtained from the ovarian tissue during an early follicular phase (days 3-5) of a spontaneous 
menstrual cycle by follicular puncture into PBS. GCs from PCOS patients were named as p-GCs and GCs from healthy controls were named as h-GCs. Cells were cultured in six-well plates $\left(1 \square 10^{6}\right.$ cells/well) using DMEM/F12 medium (HyClone, Logan, UT, USA) supplemented with 2 mM L-glutamine (Sigma-Aldrich, St. Louis, MO, USA), 10\% fetal bovine serum (FBS; Gibco, Carlsbad, CA, USA) and 1\% antibiotics (100 U/ml penicillin and $100 \mu \mathrm{g} / \mathrm{ml}$ streptomycin; Sigma-Aldrich) at $37^{\circ} \mathrm{C}$ with $5 \% \mathrm{CO}_{2}$. GCs $\left(2 \times 10^{5}\right.$ per well $)$ were seeded in 6-well plates and treated with testosterone at the concentration of $0,0.1,10 \mathrm{nM}$ for indicated times to examine the effects of testosterone on the expressions of miR-16 and PDCD4, cell proliferation, cell cycle and apoptosis in GCs.

\section{RNA extraction and quantitative real-time PCR ( $q R T-P C R)$}

Whole blood samples were collected from the antecubital area of women during an early follicular phase of a spontaneous menstrual cycle. Total RNA was isolated from ovarian cortex tissues, whole blood samples or GCs using Trizol Reagent (Invitrogen) according to the manufacturer's instructions. The first strand of cDNAs for miR-16 and PDCD4 mRNA was synthesized using the Taqman Advanced microRNA reverse-transcription kit (Applied Biosystems Inc., Foster City, CA, USA) or PrimeScript RT Reagent Kit (TaKaRa, Tokyo, Japan), respectively. The expression levels of miR-16 and PDCD4 mRNA were detected by TaqMan Universal PCR Master Mix 2X (Applied Biosystems) and the SYBR Premix Ex Taq (TaKaRa) on a 7500 Fast Real-Time PCR system (Applied Biosystems), respectively. Small nuclear U6 RNA and GAPDH were used as the internal control of miRNA and mRNA. The primer sequences for amplification were as below: miR-16 forward, 5'-TAGCAGCACGTAAATATTGGCG-3', reverse, 5'-TGCGTGTCGTGGAGTC-3'; U6 forward, 5'-GCTTCGGCAGCACATATACTAAAAT-3', reverse, 5'-CGCTTCACGAATTTGCGTGTCAT-3'; PDCD4 forward, 5'-TGGATTAACTGTGCCAACCA-3', reverse 5'-TCTCAAATGCCCTTTCATCC-3'; GAPDH forward 5'-TATGATGATATCAAGAGGGTAGT-3', reverse 5'-TGTATCCAAACTCATTGTCATAC-3'.

\section{Cell transfection}

miR-16 mimics (miR-16) and corresponding scrambled control (miR-control), miR-16 inhibitor (antimiR-16) and corresponding scrambled control (anti-miR-control), siRNA against PDCD4 (si-PDCD4) and its scrambled control (si-control) were purchased from GenePharma Co. Ltd. (Shanghai, China). PDCD4 cDNA inserts were obtained by qRT-PCR and PDCD4-overexpression vector was achieved by cloning PDCD4 sequence into the HindIII and BamHI restriction sites of pcDNA empty vector (Promega Corp., Madison, WI, USA), named as pcDNA-PDCD4. GCs were seeded into 24 -well plates at a density of $2 \times 10^{5}$ per well one day before transfection. To manipulate the expressions of miR-16 or PDCD4, GCs were transfected with miRNA mimics, miRNA inhibitors, plasmids or siRNAs using Lipofectamine 2000 reagent (Invitrogen, Carlsbad, CA, USA). Cells were collected $48 \mathrm{~h}$ posttransfection for further analysis.

\section{Lentivirus construction, virus packaging, titration and infection}

The required oligonucleotide fragments of miR-16 or anti-miR-16 expression vectors were synthesized by PCR and inserted into ScaI and Xhol restriction sites of eukaryotic expression vector pEGFP-C3 vector (BD Biosciences, San Jose, CA, USA) to construct pEGFP-C3-miR-16 and pEGFP-C3-anti-miR-16, which was confirmed by sequence analysis. The pEGFP-C3 vectors containing the corresponding miR-control or antimiR-control scrambled sequences were used as the controls. pEGFP-C3-miR-16 and pEGFP-C3-anti-miR-16 and pLL3.7 were digested using NheI and EcoRI enzymes, ligated and transformed into Top10 to construct lentiviral vectors pLL3.7-miRNA-16 and pLL3.7-anti-miR-16, which was confirmed by XhoI digestion. These constructed lentivirus plasmids were cotransfected into 293T cells together with the plasmids pHelper1.0 and pHelper 2.0 (GenePharma Co. Ltd.) that contain the elements required for virus packaging, using Lipofectamine 2000 (Invitrogen). The culture supernatants containing lentivirus were collected and concentrated by ultracentrifugation, and the viral titer determination was performed by double dilution method. P-GCs were incubated with culture medium overnight and the culture medium was replaced with viral supernatant at an appropriate titer $(1.5 \mathrm{~mL} /$ well $)$ on the day of injection. After $48 \mathrm{~h}$, the infected cells were subjected to screening with puromycin for 7 days to obtain the stable lentivirus-transfected cells.

Western blot

Total proteins were extracted from ovarian cortex tissues and cultured GCs by modified radioimmunoprecipitation assay (RIPA) buffer (Thermo Scientific, Rockford, IL, USA). The protein 


\section{Cellular Physiology Cell Physiol Biochem 2018;48:670-682 \begin{tabular}{l|l|l} 
and Biochemistry 10.1159/000491894 & $\begin{array}{l}\text { D } 2018 \text { The Author(s). Published by S. Karger AG, Basel } \\
\text { www.karger.com/cpb }\end{array}$
\end{tabular}}

Fu et al.: Role of miR-16 in Polycystic Ovarian Syndrome

samples $(20 \mu \mathrm{g})$ were subjected to $10 \%$ sodium dodecyl sulfate polyacrylamide gel electrophoresis (SDSPAGE) and then transferred onto a polyvinylidene difluoride (PVDF) membrane (Bio-Rad, Hercules, CA, USA). Subsequently, the membranes were blocked with $5 \%$ non-fat milk in TBST buffer for $2 \mathrm{~h}$ at $37^{\circ} \mathrm{C}$, probed with primary antibodies against PDCD4 (1:500, Santa Cruz Biotechnology, Santa Cruz, CA, USA), proliferating cell nuclear antigen (PCNA) (1:100, Santa Cruz Biotechnology), caspase-3 (1:500, Santa Cruz Biotechnology) and $\beta$-actin (1:5000, Santa Cruz Biotechnology) overnight at $4^{\circ} \mathrm{C}$, followed by incubation with goat anti-rabbit IgG labeled with horseradish peroxidase (1:2000, Abcam, Cambridge, MA, USA) for $1 \mathrm{~h}$ at room temperature. The protein signals were visualized using ECL detection reagent (Millipore, Billerica, MA, USA) and quantified by the Image Quant software (Molecular Dynamics, Sunnyvale, CA, USA).

\section{MTT assay}

Cell proliferation was examined by MTT assay according to the reported method [19]. GCs were plated in 96-well plates (Corning Costar Co., Corning, NY, USA) at a concentration of 4, 000 cells/well and transfected with indicated oligonucleotides or plasmids. At $24 \mathrm{~h}, 48 \mathrm{~h}, 72 \mathrm{~h}$ post-transfection, $20 \mu \mathrm{l}$ of MTT $(5 \mathrm{mg} / \mathrm{ml})$ was added to each well and incubated for another $4 \mathrm{~h}$. The supernatants were then discarded by centrifugation (1, $000 \times \mathrm{g}, 5 \mathrm{~min}$, room temperature) and $100 \mu \mathrm{l}$ of dimethyl sulfoxide (DMSO) was added to each well to dissolve the formazan product. Absorbance at a wavelength of $490 \mathrm{~nm}$ was measured using an ELx808 plate reader (BioTek Instruments, Inc., Winooski, VT, USA).

\section{Luciferase reporter assay}

The full-length of 3'UTR PDCD4 containing the predicted wild-type or mutated miR-16 binding sites were amplified by PCR and cloned into the downstream of the firefly luciferase reporter gene in pMIR (Ambion, Austin, TX, USA) vectors to generate recombinant vectors PDCD4-wt and PDCD4-mut. The constructed luciferase reporter plasmids PDCD4-wt or PDCD4-mut were cotransfected with miR-16 or miRcontrol into p-GCs, or cotransfected with anti-miR-16 or anti-miR-control into h-GCs using Lipofectamine 2000 reagent (Invitrogen). At $48 \mathrm{~h}$ after transfection, the luciferase activity was determined using the Dual-Luciferase Reporter Assay system (Promega). The Renilla luciferase activity was regarded as the normalization.

\section{Flow cytometry analysis}

GCs transfected with indicated oligonucleotides or plasmids were seeded into 6-well plates and cultured for $48 \mathrm{~h}$. For cell cycle analysis, the cells were harvested by trypsinization, washed 3 times with PBS buffer and fixed with $75 \%$ ethanol at $-20^{\circ} \mathrm{C}$ overnight. Then the fixed cells were incubated with $0.5 \mu \mathrm{g} / \mathrm{ml}$ RNase A (Thermo Fisher Scientific Inc., Wilmington, MA, USA) and $100 \mu \mathrm{g} / \mathrm{ml}$ propidium iodide (PI) for 30 $\mathrm{min}$. For apoptosis analysis, the apoptotic cells were detected with an Annexin V-fluorescein isothiocyanate/ propidium iodide apoptosis detection kit (Abcam, Cambridge, MA, USA). Both cell cycle and apoptosis were analyzed using the FACScan flow cytometry system (Becton Dickinson, San Jose, CA, USA) equipped with CellQuest software (Becton Dickinson, Mountain View, CA, USA).

\section{RNA binding protein immunoprecipitation (RIP) assay}

RIP was performed using the EZ-Magna RIP kit (Millipore). Briefly, GCs at $80-90 \%$ confluency were lysed in complete RIP lysis buffer. The whole cell extract (100 $\mu$ l) was incubated with RIP buffer containing magnetic beads conjugated with human anti-Argonaute2 (anti-Ago2) antibody (Abcam) or normal mouse IgG (Millipore). IgG was used as a negative control. Then, the samples were incubated with proteinase $\mathrm{K}$ for $20 \mathrm{~min}$ at $55^{\circ} \mathrm{C}$ to digest the proteins. Finally, the co-precipitated RNAs were isolated and subjected to qRTPCR analysis.

\section{Animals and experimental protocols}

The animal protocols were performed in accordance with the criteria for the Care and Use of Laboratory Animals and approved by the Ethics Committee of Zhujiang Hospital of Southern Medical University. 84 female Wistar rats (90 days old, weighing $160 \pm 20$ g) (Harlan, Udine, Italy) were kept in a standard specific pathogen-free environment with desired temperature of $20-24^{\circ} \mathrm{C}$ in an alternating 12 -h light/dark cycles and fed with free access to food and water. Rats were divided into 5 group including sham group, control PCOS and 4 experimental PCOS groups. The rats in PCOS group were administrated with letrozole 
(dissolved in carboxymethyl cellulose) at the dose of $1 \mathrm{mg} / \mathrm{kg}$ for 23 days. The rats in sham group were administered with the equivalent amount of carboxymethyl cellulose for 23 days. The rats in experimental PCOS groups received subcutaneous injection of $1 \times 10^{7} \mathrm{p}$-GCs cells stably transfected with lenti-miR-16, lenti-miR-control, lenti-anti-miR-16, or lenti-anti-miR-control into the left flanks for 5 weeks, followed by letrozole administration. At 5 weeks after inoculation, the mice were killed and freshly dissected ovaries were subjected to qRT-PCR and western blot.

\section{Measurement of blood levels of steroids}

After $10 \mathrm{~h}$ of fasting, blood samples were collected in sterile bottles. The serum samples were separated by centrifugation at $6,000 \mathrm{rpm}$ for five min and stored at $-20^{\circ} \mathrm{C}$ prior to analysis. The serum levels of folliclestimulating hormone $(\mathrm{FSH})$, luteinizing hormone $(\mathrm{LH})$, estradiol $\left(\mathrm{E}_{2}\right)$, and testosterone were measured using the commercial Enzyme-Linked Immunosorbent Assay (ELISA) Kits (Yanhui, Shanghai, China).

\section{Statistical analysis}

Results of experiments were presented as mean \pm standard deviation (SD) from at least three repeated experiments. The statistical analyses were performed using SPSS 17.0 (IBM, Armonk, NY, USA). The differences between groups were calculated using one-way ANOVA or Student's $t$-test. Differences were considered statistically significant at $P<0.05$.

\section{Results}

\section{miR-16 level was down-regulated in PCOS patients}

The expression of miR-16 in ovarian cortex tissues and serum of women with or without PCOS was firstly examined by qRT-PCR. As compared with healthy controls, miR-16 level was significantly lowered in both ovarian cortex tissues (Fig. 1A) and serum (Fig. 1B) of PCOS patients. The clinical characteristics of PCOS patients and healthy controls are shown in Table 1.

PDCD4 expression was up-regulated in ovarian cortex tissues of PCOS patients

We further explored the protein level of PDCD4 in ovarian cortex tissues of women with or without PCOS. As shown in Fig. 2A and 2B, an obvious increase of PDCD4 level was observed in ovarian cortex tissues of PCOS patients compared with that in healthy controls, as demonstrated by western blot.

miR-16 overexpression stimulated cell growth and decreased apoptosis in $p$-GCs

In view of the aberrant expression of miR-16 in ovarian cortex tissues and serum of PCOS patients, we then supposed
Table 1. Comparison of clinical features between PCOS patients and healthy controls. BMI, body mass index; FSH, follicle-stimulating hormone; $\mathrm{LH}$, luteinizing hormone

\begin{tabular}{llll}
\hline & Control $(\mathrm{n}=19)$ & PCOS $(\mathrm{n}=19)$ & $P$ value \\
\hline Age $($ year) & $27.8 \pm 3.7$ & $26.9 \pm 3.2$ & 0.731 \\
BMI $\left(\mathrm{kg} / \mathrm{m}^{2}\right)$ & $21.8 \pm 2.9$ & $24.1 \pm 3.4$ & 0.018 \\
FSH $(\mathrm{mIU} / \mathrm{mL})$ & $6.6 \pm 1.9$ & $5.7 \pm 1.3$ & 0.012 \\
LH (mIU/mL) & $6.8 \pm 3.2$ & $9.8 \pm 4.7$ & 0.037 \\
Prolactin $(\mathrm{ng} / \mathrm{mL})$ & $11.9 \pm 4.5$ & $36.9 \pm 7.3$ & 0.031 \\
Estradiol $(\mathrm{pg} / \mathrm{mL})$ & $31.8 \pm 2.9$ & $76.1 \pm 7.1$ & 0.027 \\
Glucose $(\mathrm{mmol} / \mathrm{mL})$ & $4.7 \pm 1.3$ & $1.5 \pm 0.1$ & 0.014 \\
Testosterone $(\mathrm{nmol} / \mathrm{mL})$ & $1.3 \pm 0.2$ & $2.1 \pm 0.3$ & 0.004 \\
Hyperandrogenemia, \% (n) & $10.5(2)$ & $52.6(10)$ & 0.005 \\
Normal androgens, \% (n) & $89.5(17)$ & $42.1(8)$ & 0.002 \\
Insulin (mU/mL) & $7.6 \pm 1.2$ & $21.8 \pm 3.5$ & 0.041 \\
Hyperinsulinism, \% (n) & $5.3(1)$ & $42.1(8)$ & 0.008 \\
Number of oocytes & $18.3 \pm 2.6$ & $8.2 \pm 2.7$ & 0.001 \\
Number of good embryos & $11.3 \pm 8.4$ & $4.3 \pm 2.6$ & 0.001 \\
\hline
\end{tabular}

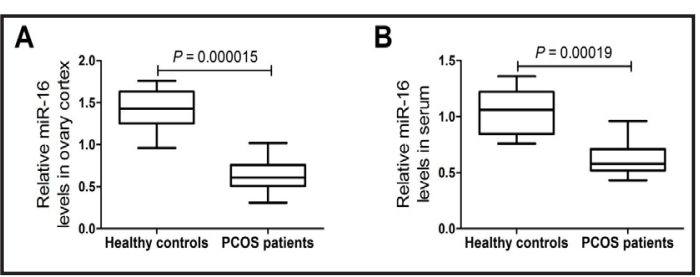

Fig. 1. miR-16 level was down-regulated in ovarian cortex tissues and serums of PCOS patients. qRT-PCR was implemented to detect the expression of miR-16 in ovarian cortex tissues (A) and serum samples (B) of PCOS patients and healthy controls. 
Fig. 2. Protein level of PDCD4 was up-regulated in ovarian cortex tissues of PCOS patients. (A) Western blot analysis of PDCD4 protein level in ovarian cortex tissues of PCOS patients and healthy controls. (B) Quantification analysis of relative protein level of PDCD4.

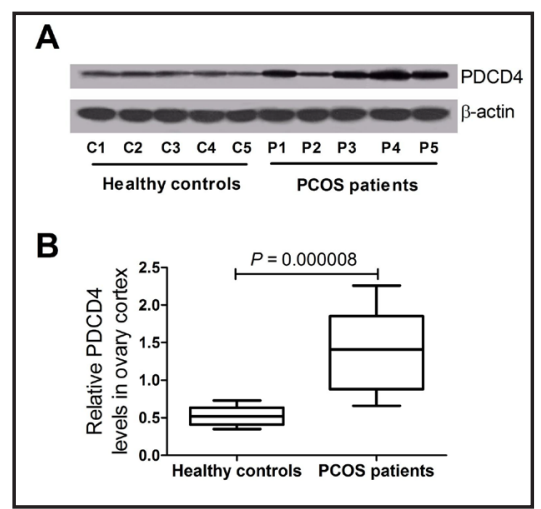

Fig. 3. miR-16 overexpression promoted growth and suppressed apoptosis in p-GCs. p-GCs were transfected with miR-16 or miRcontrol, followed by detection of miR-16 expression by qRT-PCR analysis (A), cell proliferation ability by MTT assay (B), cell cycle distribution and apoptosis by flow cytometry (C and D). h-GCs were introduced with anti-miR-control or anti-miR-16, followed by measurement of miR-16 expression by qRT-PCR analysis (E), cell proliferation ability by MTT assay (F), cell cycle distribution and apoptosis by flow cytometry ( $\mathrm{G}$ and $\mathrm{H}$ ). ${ }^{*} \mathrm{P}<0.05$.

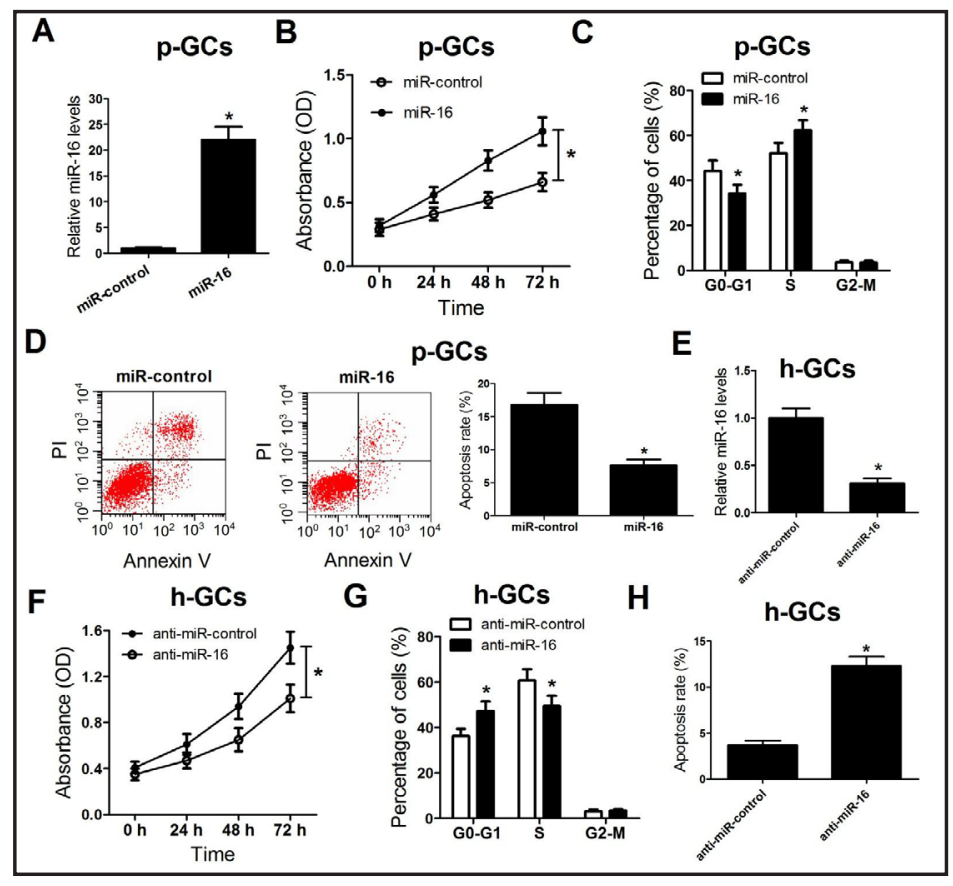

that miR-16 may be involved in the pathogenesis of PCOS. To confirm the hypothesis, we successfully established miR-16-overexpressing p-GCs by transfecting with miR-16 mimics (Fig. 3A), and miR-16-downregulated h-GCs by introducing with anti-miR-16 (Fig. 3E). MTT assay was applied to determine the impact of miR-16 overexpression or downregulation on GCs proliferation. The results showed that forced expression of miR-16 triggered a marked promotion of cell proliferation compared to miR-control group in p-GCs (Fig. 3B), while miR-16 knockdown led to a significant suppression of cell proliferation compared with antimiR-control group in h-GCs (Fig. 3F). Next, we analyzed the effects of miR-16 on cell cycle distribution and apoptosis by flow cytometry in p-GCs and h-GCs. As illustrated in Fig. 3C, miR-16 restoration in p-GCs resulted in a significant decrease of cell populations at G0-G1 phase and an obvious elevation of cell proportion at $\mathrm{S}$ phase, compared with control group. However, miR-16 down-regulation in h-GCs increased the percentage of cells at G0-G1 phase, while reduced the distribution of cells at S stage (Fig. 3G). Meanwhile, a remarkable decline of apoptosis was observed in miR-16-overexpressing p-GCs relative to miR-control group (Fig. 3D), while a dramatic rise of apoptosis was found in miR-16-downregulated h-GCs versus anti-miR-control group (Fig. $3 \mathrm{H}$ ). Collectively, miR-16 overexpression contributed to proliferation and inhibited apoptosis in p-GCs. 
Fig. 4. miR-16 suppressed PDCD4 expression by targeting 3'UTR of PDCD4 in p-GCs and h-GCs. (A) Schematic description of the wildtype and mutant miR-16 binding sites in the 3'UTR of PDCD4. (B) Analysis of relative luciferase activity was performed at $48 \mathrm{~h}$ after p-GCs were cotransfected with PDCD4-wt or PDCD4-mut reporter and miR-16 or miR-control. (C) Endogenous interaction between miR-16 and PDCD4 in p-GCs was confirmed by RIP assay with Ago2 antibody, followed by qRT-PCR analysis of PDCD4 mRNA and miR16. (D and E) PDCD4 expression at mRNA and protein levels was assessed by qRT-PCR and western blot analysis in p-GCs after

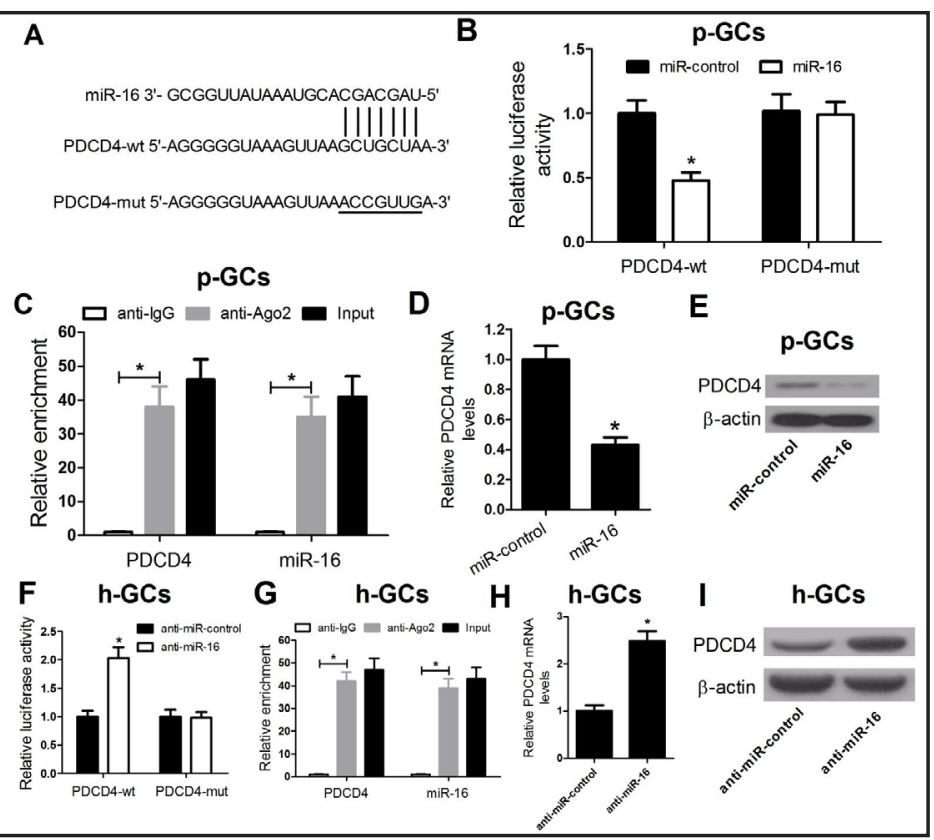
transfection with miR-16 mimic or miR-control. (F) The relative luciferase activity was measured at $48 \mathrm{~h}$ after h-GCs were cotransfected with PDCD4-wt or PDCD4-mut reporter and anti-miR-16 or anti-miR-control. (G) RIP assay was applied to verify the interaction between miR-16 and PDCD4 in h-GCs using Ago2 antibody. (H and I) qRT-PCR and western blot analysis were used to evaluate PDCD4 expression at mRNA and protein levels in h-GCs after introduction with anti-miR-16 or anti-miR-control. ${ }^{*} \mathrm{P}<0.05$.

\section{PDCD4 was a direct target of miR-16 in $p$-GCs and $h-G C s$}

Previous studies have demonstrated that PDCD4 was a target of miR-16 in atherosclerosis and miR-16 targeted PDCD4 to suppress the activation of inflammatory macrophages [20]. Thus, we further explored whether PDCD4 was a putative target of miR-16 in p-GCs and h-GCs. Bioinformatics prediction based on TargetScan displayed that there existed a binding sequence of miR-16 in the 3'UTR of PDCD4 (Fig. 4A). The luciferase activity of reporter with wild-type 3'UTR was exceptionally suppressed in miR-16-transfected (Fig. 4B) p-GCs but was significantly promoted in anti-miR-16-transfected h-GCs (Fig. 4F), while no obvious change in the luciferase activity of reporters with mutant 3'UTR of PDCD4 was observed in miR-16-transfected p-GCs and anti-miR-16-transfected h-GCs. It is well established that miRNAs directly bind to the mRNA molecules by forming miRNA ribonucleoprotein complexes (miRNPs) with Ago2, the key component of the RNA-induced silencing complex (RISC), resulting in translational repression and/or mRNA degradation [21, 22]. To further verify the direct interaction between miR-16 and PDCD4, we tested miR-16-mediated binding of RISC to PDCD4 mRNA using RIP assay with antibody against Ago2. The expression levels of miR-16 and PDCD4 mRNA were detected by qRT-PCR. As expected, expressions of miR-16 and PDCD4 mRNA were both significantly enriched in Ago2 complexes purified from both p-GCs (Fig. 4C) and h-GCs (Fig. 4G) versus negative control group, suggesting the endogenous binding between miR-16 and PDCD4. As demonstrated by qRT-PCR and western blot, transfection with miR-16 mimics evidently repressed the expression of PDCD4 at mRNA (Fig. 4D) and protein levels (Fig. 4E) in p-GCs. On the contrary, introduction with anti-miR-16 elicited a prominent increase of PDCD4 expression at mRNA (Fig. 4H) and protein levels (Fig. 4I) in h-GCs. Together, these results indicated that miR-16 directly down-regulated PDCD4 expression by binding to its 3'UTR. 
Fig. 5. miR-16 overexpression accelerated cell proliferation and decreased apoptosis by targeting PDCD4 in p-GCs. p-GCs were transfected with miR-control, miR-16, miR-16+Vector, or miR16+pcDNA-PDCD4, while h-GCs were introduced with antimiR-control, anti-miR-16, antimiR-16+si-control, or anti-miRcontrol+si-PDCD4. MTT assay was performed to determine cell viability at $24 \mathrm{~h}, 48 \mathrm{~h}$, and 72 $\mathrm{h}$ in transfected p-GCs (A) and h-GCs (D). Cell cycle distribution was detected by flow cytometry in transfected p-GCs (B) and h-GCs (E). The apoptotic rate in transfected p-GCs (C) and h-GCs (F) was examined by flow cytometry. $* \mathrm{P}<0.05$.

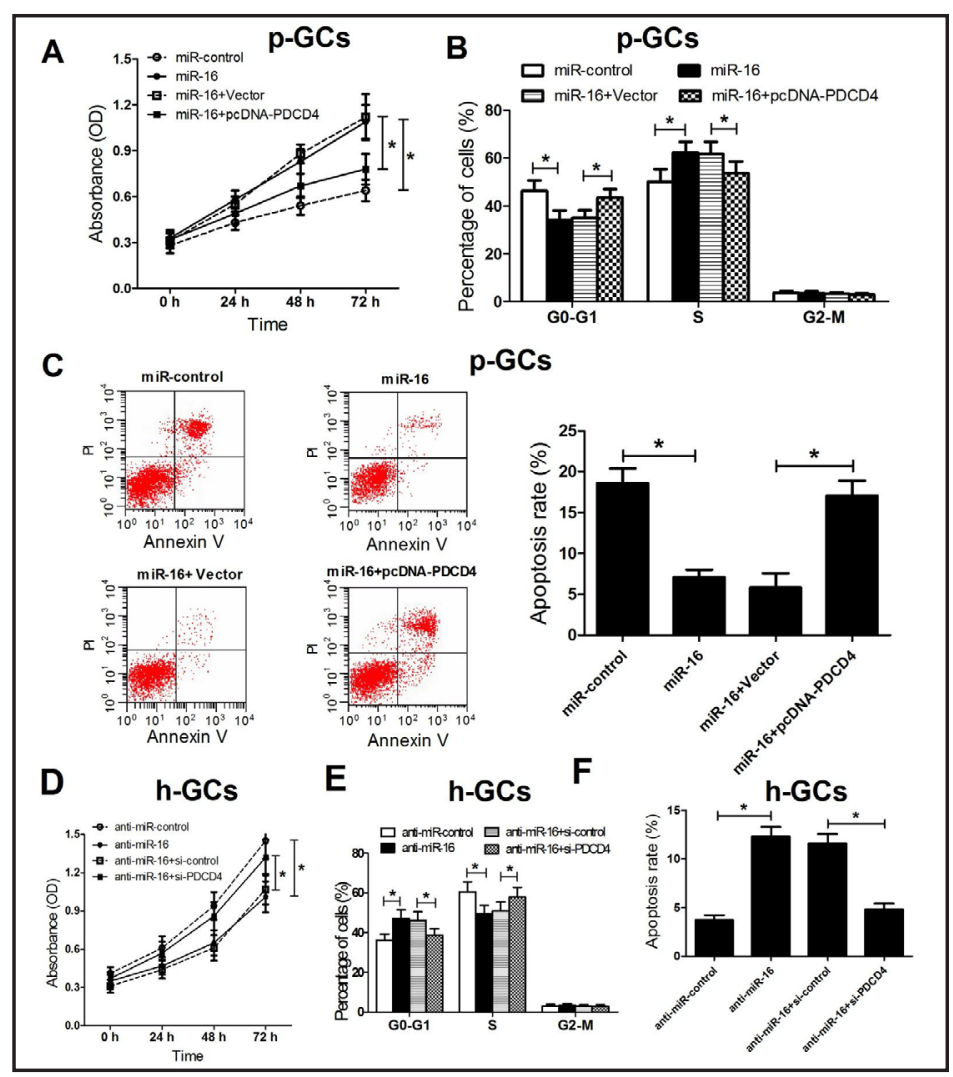

PDCD4 overexpression abated the effect of miR-16 on cell proliferation and apoptosis in $p$-GCS

To explore whether PDCD4 was involved in the biological roles of miR-16 in p-GCs and h-GCs, miR-16-overexpressing p-GCs were transfected with pcDNA-PDCD4 and miR16-downregulated h-GCs were introduced with si-PDCD4. As demonstrated by MTT assay, cell proliferation was obviously improved in miR-16-transfected p-GCs, while PDCD4 overexpression remarkedly attenuated the proliferation promotion induced by miR-16 overexpression (Fig. 5A). In contrast, PDCD4 knockdown partially reversed the inhibitory effect of anti-miR-16 on the proliferation ability in h-GCs (Fig. 5D). In addition, miR-16induced cell cycle progression was partly abrogated by overexpression of PDCD 4 in p-GCs (Fig. 5B). On the contrary, cell cycle arrest triggered by anti-miR-16 was undermined by PDCD 4 knockdown (Fig. 5E). Furthermore, miR-16-overexpressing p-GCs presented a remarkable reduction in the percentage of apoptotic cells relative to miR-control group, while this effect was greatly counteracted following PDCD4 overexpression (Fig. 5C). Conversely, PDCD4 knockdown dramatically mitigated the promotive effect of anti-miR-16 on the apoptosis of h-GCs (Fig. 5F). All these results suggested that miR-16 overexpression boosted cell growth and diminished apoptosis by targeting PDCD4 in p-GCs.

Testosterone down-regulated miR-16, blocked cell growth and induced apoptosis in p-GCs

Since PCOS is frequently characterized by hyperandrogenemia [23], we supposed that the expression difference of miR-16 in our findings was induced by the increase of testosterone. We firstly explored the effect of testosterone on the expression level of miR-16 and PDCD4 by qRT-PCR or western blot in $p$-GCs treated with $0.1 \mathrm{nM}$ or $10 \mathrm{nM}$ testosterone for $24 \mathrm{~h}$. miR-16 expression was gradually decreased (Fig. 6A), and PDCD4 expression at mRNA and protein level was progressively improved (Fig. 6B and 6C) along with the increase of testosterone concentration compared with the control group. Moreover, we found that high concentration of testosterone $(10 \mathrm{nM})$ remarkably suppressed cell proliferation (Fig. 
6D) and induced cell cycle arrest at G0-G1 phase (Fig. 6E) in p-GCs. Moreover, the apoptotic rate of p-GCs was significantly enhanced after testosterone treatment (Fig. 6F). Therefore, these findings demonstrated that testosterone inhibited cell growth and promoted apoptosis possibly by down-regulating miR-16 and up-regulating PDCD4.

\section{miR-16 overexpression ameliorated PCOS in vivo by regulating PDCD4}

To confirm the biological role and molecular mechanism of miR-16 in PCOS in vivo, we established an animal model of PCOS by treating rats with letrozole. The serum levels of FSH, $\mathrm{LH}, \mathrm{E}_{2}$, and testosterone were measured using ELISA. As shown in Fig. 7A-D, the serum levels of FSH, LH, and testosterone were significantly higher, while E2 level was dramatically lower in PCOS group than that in sham group, suggesting the successful establishment of PCOS model. The mice were killed after 5 weeks of inoculation and freshly dissected ovaries were subjected to qRT-PCR and western blot. As expected, miR-16 expression was remarkably increased in lenti-miR-16-injected group and was notably decreased in lenti-anti-miR-16-

Fig. 6. Testosterone downregulated miR-16, impeded $\mathrm{p}-\mathrm{GCs}$ growth and induced apoptosis. p-GCs were treated with $0.1 \mathrm{nM}$ or $10 \mathrm{nM}$ testosterone for $24 \mathrm{~h}$, followed by detection of miR-16 expression (A), as well as PDCD4 mRNA and protein levels (B and C). (D) Cell viability was detected by MTT assay at $24 \mathrm{~h}, 48 \mathrm{~h}$, and $72 \mathrm{~h}$ in GCs treated with $10 \mathrm{nM}$ testosterone. (E) The cell numbers were counted by flow cytometry with PI staining according to the DNA content of G0-G1, S, and G2-M phases after p-GCs were exposed to $10 \mathrm{nM}$ testosterone for $48 \mathrm{~h}$. (F) Cell apoptosis analysis after p-GCs were treated with $10 \mathrm{nM}$ testosterone for $48 \mathrm{~h} .{ }^{*} \mathrm{P}<0.05$.



Fig. 7. miR-16 overexpression alleviated PCOS in vivo by regulating $\mathrm{PDCD} 4$. The serum levels of FSH (A), LH (B), E2 (C), and testosterone (D) from sham and PCOS groups were measured by ELISA. The expressions of miR-16 (E) and PDCD4 mRNA (F) in control PCOS group and 4 experimental PCOS groups were detected by qRT-PCR. (G) The protein levels of PDCD4, PCNA and caspase-3 in control PCOS group and 4 experimental PCOS groups were assessed by western blot. The serum levels of FSH $(\mathrm{H}), \mathrm{LH}$ (I), E2 (J), and testosterone (K) from control PCOS group and 4 experimental PCOS groups were evaluated by ELISA. ${ }^{*} \mathrm{P}<0.05$.

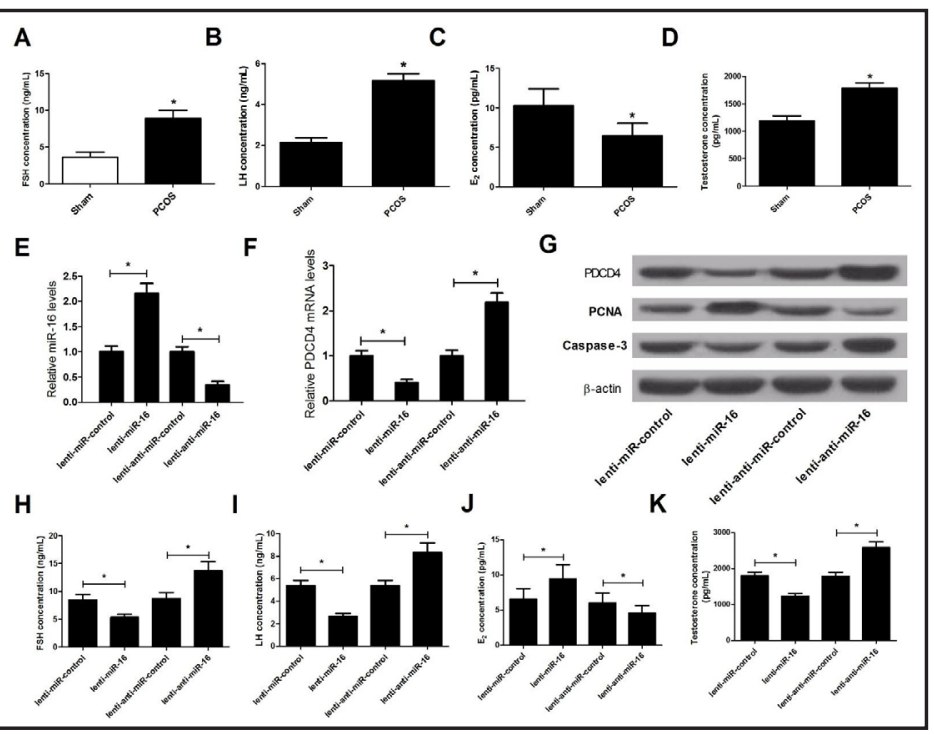


injected group compared with matched controls (Fig. 7E). In contrast, PDCD4 mRNA and protein expressions were obviously reduced in lenti-miR-16-injected group and dramatically enhanced in lenti-anti-miR-16-injected group (Fig. 7F and 7G). Additionally, western blot analysis disclosed that ectopic expression of miR-16 in p-GCs led to an obvious increase of PCNA protein level and an apparent decrease of caspase-3 level, while miR-16 downregulation elicited the opposite effects in the animal model of PCOS (Fig. 7G), suggesting that miR-16 overexpression promoted p-GCs cell growth and inhibited apoptosis in PCOS in vivo. Furthermore, ELISA results revealed that enforced expression of miR-16 in p-GCs restrained the serum levels of FSH (Fig. 7H), LH (Fig. 7I), and testosterone (Fig. 7K), and elevated $\mathrm{E}_{2}$ level (Fig. 7J) in PCOS in vivo compared with that in lenti-miR-control-injected group. However, miR-16 inhibition exerted the contrary effects on the serum levels of FSH, LH, $\mathrm{E}_{2}$ and testosterone (Fig. $7 \mathrm{H}-7 \mathrm{~K}$ ). Together, these data suggested that miR-16 overexpression alleviated PCOS in vivo by targeting PDCD4.

\section{Discussion}

During the development of ovarian follicular, degenerative atresia happened in over $99 \%$ of the follicles, and only a few follicles are able to ovulate. Apoptosis of GCs have been demonstrated to be involved in follicular atresia [24]. Increasing evidence suggests many miRNAs participate in the proliferation, apoptosis and differentiation of GCs in the ovary [25] and are involved in the pathological progression of PCOS [26]. For instance, miR-320, transactivated by miR-383, was up-regulated in GCs and follicular fluid of PCOS patients, and miR-320 overexpression suppressed GC production and steroid production by regulating transcriptional factor E2F1 and steroidogenic factor 1 (SF-1) [27]. miR-92a was down-regulated in atretic porcine follicles, and ectopic expression of miR-92a suppressed GC apoptosis through modulating Smad7 gene [28]. In contrast, miR-145 expression was reported to be decreased in human p-GCs, and miR-145 mimics hindered cell proliferation and induced apoptosis by inhibiting insulin receptor substrate 1 (IRS1) expression [29]. Increased expression of miR-93 was demonstrated to promote GC proliferation and cell cycle progression by targeting cyclin dependent kinase inhibitor 1A (CDKN1A) in PCOS [30]. This study was designed to explore the functions and possible molecular basis of miR-16 in GCs dysfunction in PCOS.

miR-16 was previously disclosed to be down-regulated in PCOS patients likely to suffer from severe ovarian hyperstimulation syndrome (OHSS) [17]. Similarly, our results manifested that miR-16 expression was lowered in the ovarian cortex tissues and serum of PCOS patients. Functional experiments further demonstrated that overexpression of miR-16 mimics facilitated cell proliferation, promoted cell cycle progression and inhibited apoptosis in p-GCs, while miR-16 knockdown blocked cell proliferation, induced cell cycle arrest and enhanced apoptosis in h-GCs. Furthermore, in vivo experiments demonstrated that miR-16 overexpression alleviated PCOS and miR-16 down-regulation aggravated PCOS in vivo. Consistently, miR-16 was previously reported to be up-regulated in renal cell carcinom, and acted as an oncogene in RCC by inducing cellular proliferation, migration and reducing apoptosis [31]. However, enforced expression of miR-16 impaired cell proliferation and promoted apoptosis in lung adenocarcinoma [32], esophageal squamous cell carcinoma [33] and breast cancer [34], suggesting that miR-16 may exert different functions in various diseases.

PDCD4, located on chromosome 10q24, was initially identified as an up-regulated gene during apoptosis [35]. PDCD4 is widely recognized as a protein translation suppressor by binding to the translation initiation factor eIF4A, inhibiting its RNA-helicase activity [36, 37]. PDCD4 has been discovered to be involved in some metabolic diseases. PDCD4 deficiency protected mice against diet-induced obesity, adipose tissue inflammation, and insulin resistance, suggesting that PDCD4 may be a potential target for the treatment of obesityassociated diseases [38]. More importantly, PDCD4 was highly expressed in PCOS patients,

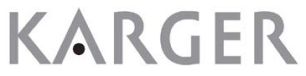


and knockdown of PDCD4 significantly suppressed apoptosis of GCs in women with PCOS [39]. In accordance with these previous studies, our study found that PDCD4 expression was up-regulated in the ovarian cortex tissues of PCOS patients. Moreover, PDCD4 was validated to be a direct target of miR-16 [20], and miR-16 inhibited PDCD4 expression in p-GCs in vivo and in vitro. Furthermore, PDCD4 overexpression abolished miR-16-induced proliferation promotion, cell cycle progression, and apoptosis inhibition in p-GCs, while PDCD4 knockdown attenuated anti-miR-16-triggered proliferation inhibition, cell cycle arrest and apoptosis promotion in h-GCs. Of note, PCOS is characterized by hyperandrogenism, which leads to PCO morphology and ovulatory dysfunction in women with PCOS [40]. Hyperandrogenism, a fundamental factor in the pathogenesis of PCOS, increases the level of androgen [41]. Our study demonstrated that testosterone inhibited cell growth and enhanced apoptosis possibly by repressing miR-16 and elevating PDCD4.

In conclusion, our study demonstrated that miR-16 level was decreased and PDCD4 expression was increased in PCOS patients. Mechanistic analysis elucidated that exogenous expression of miR-16 accelerated cell proliferation, promoted cell cycle progression and decreased apoptosis by targeting PDCD4 in p-GCs. Furthermore, we found that testosterone induced miR-16 down-regulation and suppressed p-GCs growth. These results contributed to a deep understanding of the molecular mechanism of GCs dysregulation in PCOS, suggesting that miR-16 may be a potential useful target for improving GCs dysfunction in PCOS.

\section{Abbreviations}

PCOS; polycystic (ovarian syndrome); p-GCs; granulosa (cells from PCOS patients); h-GCs; granulosa (cells from healthy controls); GCs; granulosa (cells); T2DM; type (2 diabetes mellitus); 3'UTR; 3' (untranslated regions); PDCD4; programmed (cell death protein 4); SDS-PAGE; sodium (dodecyl sulfate polyacrylamide gel electrophoresis); RIPA; radioimmunoprecipitation (assay); PVDF; polyvinylidene (difluoride); DMSO; dimethyl (sulfoxide); RISC; RNA-induced (silencing complex); SF-1; steroidogenic factor (1); CDKN1A; cyclin (dependent kinase inhibitor 1A); IRS1; insulin (receptor substrate 1).

\section{Acknowledgements}

Thanks to all participants involved in this research.

\section{Disclosure Statement}

The authors of this paper state that they have no conflict of interests to declare.

\section{References}

1 Teede HJ, Joham AE, Paul E, Moran LJ, Loxton D, Jolley D, Lombard C: Longitudinal weight gain in women identified with polycystic ovary syndrome: results of an observational study in young women. Obesity (Silver Spring) 2013;21:1526-1532.

-2 The Rotterdam ESHRE/ASRM-Sponsored PCOS Consensus Workshop Group: Revised 2003 consensus on diagnostic criteria and long-term health risks related to polycystic ovary syndrome (PCOS). Fertil Steril 2004;81:19-25.

-3 Diamanti-Kandarakis E, Dunaif A: Insulin resistance and the polycystic ovary syndrome revisited: an update on mechanisms and implications. Endocr Rev 2012;33:981-1030. 


\section{Cellular Physiology Cell Physiol Biochem 2018;48:670-682 \begin{tabular}{ll|l} 
and Biochemistry Published online: July 19, 2018 & $\begin{array}{l}\text { C } 2018 \text { The Author(s). Published by S. Karger AG, Basel } \\
\text { www.karger.com/cpb }\end{array}$ \\
\hline
\end{tabular}}

- 4 Krishna MB,Joseph A,Thomas PL, Dsilva B, Pillai SM, Laloraya M: Impaired Arginine Metabolism Coupled to a Defective Redox Conduit Contributes to Low Plasma Nitric Oxide in Polycystic Ovary Syndrome. Cell Physiol Biochem 2017;43:1880-1892.

5 Zuo T, Zhu MH, Xu WM, Wang ZX, Song H: Iridoids with Genipin Stem Nucleus Inhibit LipopolysaccharideInduced Inflammation and Oxidative Stress by Blocking the NF- $\kappa B$ Pathway in Polycystic Ovary Syndrome. Cell Physiol Biochem 2017;43:1855-1865.

6 Pasquali R, Stener-Victorin E, Yildiz BO, Duleba AJ, Hoeger K, Mason H, Homburg R, Hickey T, Franks S, Tapanainen JS, Balen A, Abbott DH, Diamanti-Kandarakis E, Legro RS: PCOS Forum: research in polycystic ovary syndrome today and tomorrow. Clin Endocrinol (Oxf) 2011;74:424-433.

-7 Das M, Djahanbakhch O, Hacihanefioglu B, Saridogan E, Ikram M, Ghali L, Raveendran M, Storey A: Granulosa cell survival and proliferation are altered in polycystic ovary syndrome. J Clin Endocrinol Metab 2008;93:881-887.

-8 Erickson GF, Magoffin DA, Garzo VG, Cheung AP, Chang RJ: Granulosa cells of polycystic ovaries: are they normal or abnormal? Hum Reprod 1992;7:293-299.

-9 Flynt AS, Lai EC: Biological principles of microRNA-mediated regulation: shared themes amid diversity. Nat Rev Genet 2008;9:831-842.

10 Xiang Y, Song Y, Li Y, Zhao D, Ma L, Tan L: miR-483 is Down-Regulated in Polycystic Ovarian Syndrome and Inhibits KGN Cell Proliferation via Targeting Insulin-Like Growth Factor 1 (IGF1). Med Sci Monit 2016;22:3383-3393.

11 Murri M, Insenser M, Fernandez-Duran E, San-Millan JL, Escobar-Morreale HF: Effects of polycystic ovary syndrome (PCOS), sex hormones, and obesity on circulating miRNA-21, miRNA-27b, miRNA-103, and miRNA-155 expression. J Clin Endocrinol Metab 2013;98:E1835-1844.

12 Sorensen AE, Udesen PB, Wissing ML, Englund AL, Dalgaard LT: MicroRNAs related to androgen metabolism and polycystic ovary syndrome. Chem Biol Interact 2016;259:8-16.

13 Humplikova L, Kollinerova S, Papajik T, Pikalova Z, Holzerova M, Prochazka V, Divoka M, Modriansky M, Indrak K, Jarosova M: Expression of miR-15a and miR-16-1 in patients with chronic lymphocytic leukemia. Biomed Pap Med Fac Univ Palacky Olomouc Czech Repub 2013;157:284-293.

14 Mobarra N, Shafiee A, Rad SM, Tasharrofi N, Soufizomorod M, Hafizi M, Movahed M, Kouhkan F, Soleimani M: Overexpression of microRNA-16 declines cellular growth, proliferation and induces apoptosis in human breast cancer cells. In vitro Cell Dev Biol Anim 2015;51:604-611

15 Bonci D, Coppola V, Musumeci M, Addario A, Giuffrida R, Memeo L, D’Urso L, Pagliuca A, Biffoni M, Labbaye C, Bartucci M, Muto G, Peschle C, De Maria R: The miR-15a-miR-16-1 cluster controls prostate cancer by targeting multiple oncogenic activities. Nat Med 2008;4:1271-1277.

-16 Bandi N, Zbinden S, Gugger M, Arnold M, Kocher V, Hasan L, Kappeler A, Brunner T, Vassella E: miR-15a and miR-16 are implicated in cell cycle regulation in a Rb-dependent manner and are frequently deleted or down-regulated in non-small cell lung cancer. Cancer Res 2009;69:5553-5559.

17 Zhao C, Liu X, Shi Z, Zhang J, Zhang J, Jia X, Ling X: Role of Serum miRNAs in the Prediction of Ovarian Hyperstimulation Syndrome in Polycystic Ovarian Syndrome Patients. Cell Physiol Biochem 2015;35:10861094.

18 Nordhoff V, Sonntag B, Von TD, Götte M, Schüring AN, Gromoll J, Redmann K, Casarini L, Simoni M: Effects of the FSH receptor gene polymorphism p.N680S on cAMP and steroid production in cultured primary human granulosa cells. Reprod Biomed Online 2011;23:196-203.

19 Mosmann T: Rapid colorimetric assay for cellular growth and survival: application to proliferation and cytotoxicity assays. J Immunol Methods 1983;65:55-63.

20 Liang X, Xu Z, Yuan M, Zhang Y, Zhao B, Wang J, Zhang A, Li G: MicroRNA-16 suppresses the activation of inflammatory macrophages in atherosclerosis by targeting PDCD4. Int J Mol Med 2016;37:967-975.

21 Chen D, Chen Z, Jin Y, Dragas D, Zhang L, Adjei BS, Wang A, Dai Y, Zhou X: MicroRNA-99 family members suppress Homeobox A1 expression in epithelial cells. Plos One 2013, 8:e80625.

22 Izaurralde E: Elucidating the temporal order of silencing. EMBO Rep 2012;13:662-663.

-23 Shahebrahimi K, Jalilian N, Bazgir N, Rezaei M: Comparison clinical and metabolic effects of metformin and pioglitazone in polycystic ovary syndrome. Indian J Endocrinol Metab 2016;20:805-809.

-24 Matsuda F, Inoue N, Manabe N, Ohkura S: Follicular growth and atresia in mammalian ovaries: regulation by survival and death of granulosa cells. J Reprod Dev 2012, 58:44-50. 


\section{Cellular Physiology Cell Physiol Biochem 2018;48:670-682 \begin{tabular}{l|l} 
DOI: 10.1159/000491894 & $\begin{array}{l}\text { O } 2018 \text { The Author(s). Published by S. Karger AG, Basel } \\
\text { www.karger.com/cpb }\end{array}$ \\
\hline
\end{tabular}}

25 Christenson LK: MicroRNA control of ovarian function. Anim Reprod 2010;7:129-133.

26 Sørensen AE, Udesen PB2, Wissing ML, Englund ALM, Dalgaard LT: MicroRNAs related to androgen metabolism and polycystic ovary syndrome. Chem Biol Interact 2016;259:8-16.

-27 Yin M, Wang X, Yao G, Lü M, Liang M, Sun Y, Sun F: Transactivation of micrornA-320 by microRNA-383 regulates granulosa cell functions by targeting E2F1 and SF-1 proteins. J Biol Chem 2014;289:1823918257.

28 Liu J, Yao W, Yao Y, Du X, Zhou J, Ma B, Liu H, Li Q, Pan Z: MiR-92a inhibits porcine ovarian granulosa cell apoptosis by targeting Smad7 gene. FEBS Lett 2014;588:4497-4503.

29 Cai G, Ma X, Chen B, Huang Y, Liu S, Yang H, Zou W: MicroRNA-145 Negatively Regulates Cell Proliferation Through Targeting IRS1 in Isolated Ovarian Granulosa Cells From Patients With Polycystic Ovary Syndrome. Reprod Sci 2017;24:902-910.

30 Jiang L, Huang J, Li L, Chen Y, Chen X, Zhao X, Yang D: MicroRNA-93 promotes ovarian granulosa cells proliferation through targeting CDKN1A in polycystic ovarian syndrome. J Clin Endocrinol Metab 2015;100:729-738.

-31 Chen D, Li Y, Yu Z, Su Z, Yu W, Li Y, Yang S, Gui Y, Ni L, Lai Y: Upregulated microRNA-16 as an oncogene in renal cell carcinoma. Mol Med Rep 2015;12:1399-1404.

32 Gu Y, Wang XD, Lu JJ, Lei YY, Zou JY, Luo HH: Effect of mir-16 on proliferation and apoptosis in human A549 lung adenocarcinoma cells. Int J Clin Exp Med 2015;8:3227-3233.

-33 Zhu Y, Xia Y, Niu H, Chen Y: MiR-16 induced the suppression of cell apoptosis while promote proliferation in esophageal squamous cell carcinoma. Cell Physiol Biochem 2014;33:1340-1348.

-34 Mobarra N, Shafiee A, Rad SM, Tasharrofi N, Soufi-Zomorod M, Hafizi M, Movahed M, Kouhkan F, Soleimani M: Overexpression of microRNA-16 declines cellular growth, proliferation and induces apoptosis in human breast cancer cells. In vitro Cell Dev Biol Anim 2015;51:604-611.

-35 Lankat-Buttgereit B, Goke R: Programmed cell death protein 4 (pdcd4): a novel target for antineoplastic therapy? Biol Cell 2003;95:515-519.

36 Yang HS, Cho MH, Zakowicz H, Hegamyer G, Sonenberg N, Colburn NH: A Novel Function of the MA-3 Domains in Transformation and Translation Suppressor Pdcd4 Is Essential for Its Binding to Eukaryotic Translation Initiation Factor 4A. Mol Cell Biol 2004;24:3894-3906.

37 Yang HS, Jansen AP, Komar AA, Zheng X, Merrick WC, Costes S, Lockett SJ, Sonenberg N, Colburn NH: The transformation suppressor Pdcd4 is a novel eukaryotic translation initiation factor 4A binding protein that inhibits translation. Mol Cell Biol 2003;23:26-37.

38 Wang Q, Dong Z, Liu X, Song X, Song Q, Shang Q, Jiang Y, Guo C, Zhang L: Programmed cell death-4 deficiency prevents diet-induced obesity, adipose tissue inflammation, and insulin resistance. Diabetes 2013;62:41324143.

39 Ding L, Gao F, Zhang M, Yan W, Tang R, Zhang C, Chen ZJ: Higher PDCD4 expression is associated with obesity, insulin resistance, lipid metabolism disorders, and granulosa cell apoptosis in polycystic ovary syndrome. Fertil Steril 2016;105:1330-1337.

40 Chen MJ, Yang WS, Chen CL, Wu MY, Yang YS, Ho HN: The relationship between anti-Mullerian hormone, androgen and insulin resistance on the number of antral follicles in women with polycystic ovary syndrome. Hum Reprod 2008;23:952-957.

41 Azziz R, Carmina E, Dewailly D, Diamanti-Kandarakis E, Escobar-Morreale HF, Futterweit W, Janssen OE, Legro RS, Norman RJ, Taylor AE, Witchel SF: Positions statement: criteria for defining polycystic ovary syndrome as a predominantly hyperandrogenic syndrome: an Androgen Excess Society guideline. J Clin Endocrinol Metab 2006;91:4237-4245. 